\title{
Arthroskopie des oberen Sprunggelenkes
}

\author{
Kai Ruße, Ralf Müller-Rath, Axel Pommer, Andreas Dávid
}

\section{Zusammenfassung}

Die Arthroskopie des oberen Sprunggelenkes hat sich in letzter Zeit als ein operatives Standardverfahren etabliert. Indikationen sind posttraumatische Schäden an Gelenkkapsel und Knorpel, wie z. B. das laterale und mediale Meniskoidsyndrom, freie Gelenkkörper sowie osteochondrale Läsionen. Darüber hinaus ist die Arthroskopie indiziert bei lang anhaltenden unklaren Beschwerden im Bereich des oberen Sprunggelenkes, deren Ursache sich der klinischen und der radiologischen, inklusive der MR tomo- graphischen Diagnostik, entziehen. Der arthroskopische Untersuchungsgang beginnt mit der Anlage des anterolateralen Zugangs. Es folgen unter Schonung der gefährdeten anatomischen Strukturen weitere definierte Zugangswege im anterioren und posterioren Gelenkbereich. Dieses standardisierte Vorgehen ermöglicht die vollständige Exploration des Gelenkes. Die therapeutischen Optionen reichen von der diagnostischen Inspektion bis hin zu aufwendigen Verfahren, wie z. B. arthroskopisch asistierten Arthrodesen.

überwiegend die Indikation zur Sprunggelenksarthroskopie mit therapeutischer Zielsetzung vorgenommen werden. Die rein „diagnostische Arthroskopie“ muss die absolute Ausnahme bleiben. Nur in sehr seltenen Fällen gelingt es nicht, durch bildgebende Verfahren eine Diagnose zu stellen.

\section{Indikationen zur Arthroskopie des Sprunggelenkes}

Die Indikation zur diagnostischen Arthroskopie ist nur dann gegeben (siehe Tab.1), wenn durch eine exakte klinische, nativradiologische oder MRT-Untersuchung, ergänzt durch andere apparative Verfahren wie Sonographie und Szintigraphie, keine eindeutige Diagnose gestellt werden kann [8]. Allerdings müssen die Ausbildung und Erfahrung des Operateurs auch eine eventuell notwendige arthroskopische oder arthroskopisch assistierte Operation erlauben.

\section{Gelenkblockaden}

Als Ursache intermittierender Gelenkblockierungen mit Bewegungseinschränkungen müssen differenzialdiagnostisch

Tab. 1 Indikationen zur OSG Arthros-
kopie.
rezidivierende, unklare Gelenk-
schmerzen
Gelenkblockaden
Knorpelläsionen
Osteochondrosis dissecans
- rheumatische Ergussbildung
- Weichteilimpingement
- fibulare Kapsel-Bandinstabilität
osteophytäres Impingement

freie Gelenkkörper, fixierte Gelenkkörper sowie Vernarbungen und Verwachsungsstränge in Betracht gezogen werden (siehe Abb.1a-c). Freie Gelenkkörper sind häufig radiologisch nicht darstellbar. Gelegentlich entziehen sie sich auch einer Darstellung im MRT. In einem solchen Fall ist die Diagnose nur durch Arthroskopie möglich.

\section{Rezidivierende Gelenkschmerzen}

Nach Verletzungen mit Beteiligung der vorderen Gelenkkapsel, nach Frakturen oder fibularen Bandverletzungen leiden viele Patienten oftmals über Jahre an persistierenden Beschwerden des oberen Sprunggelenkes [7]. Als diagnostische Untersuchungsmethode wird immer wieder allein auf das Röntgenbild gesetzt. Die Darstellung der vermeintlichen schmerzauslösenden Strukturen ist hiermit aber nicht immer möglich. Aus diesem Grund wird dem Schmerz des Patienten häufig nicht adäquat Rechnung getragen. Als Ursache der Beschwerden können verschiedene Arten von Synovialitiden sein [5]. Darüber hinaus können ligamentäre Impingementsyndrome [10] (laterales oder mediales Meniskoidsyndrom) (siehe Abb.1a-c), intraartikuläre Verwachsungen nach Kapselverletzungen oder ausgeprägte Kapselfibrosen als Folge von Sprunggelenksfrakturen in Frage kommen [3]. 

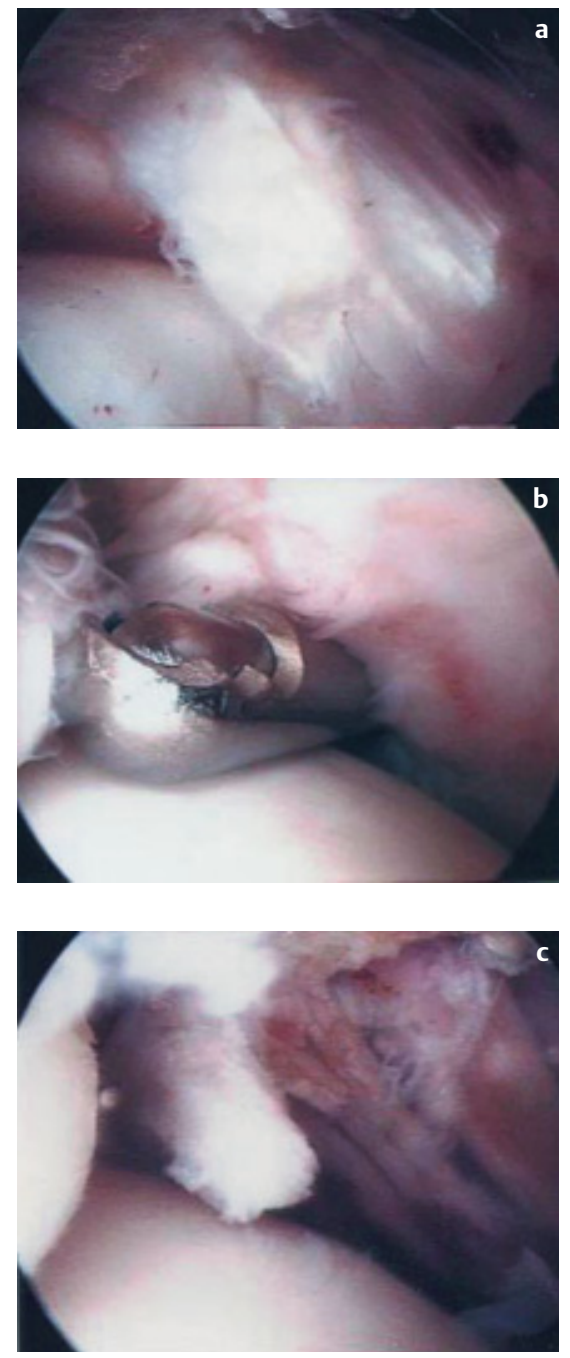

Abb.1a-c Verdickter, bindegewebiger Narbenstrang, der die laterale Taluskante überdeckt im Sinne eines "Meniskoid-Syndroms“ (a). Resektion mit dem Arthroshaver (b). Zustand nach Resektion (c).

\section{Osteochondrosis dissecans}

Die Ausdehnung eines Osteochondrosis dissecans-Herdes (siehe Abb. 2a-c), sowie das Stadium sollten vor der Arthroskopie durch radiologische, MR-tomographische oder ergänzend auch szintigraphische Untersuchungen dargestellt werden [7]. Nach Beurteilung des Osteochondrosis-dissecans-Herdes erfolgt in Abhängigkeit des Stadiums der therapeutische Eingriff. Das Spektrum reicht von der arthroskopisch kontrollierten subchondralen Anbohrung (siehe $\mathbf{A b b} \mathbf{3} \mathbf{a}-\mathbf{b}$ ) bis hin zur offenen Knorpel/Knochentransplantation mit Innenknöchelosteotomie bei größerer Ausdehnung der Herde in Talus und Tibia.
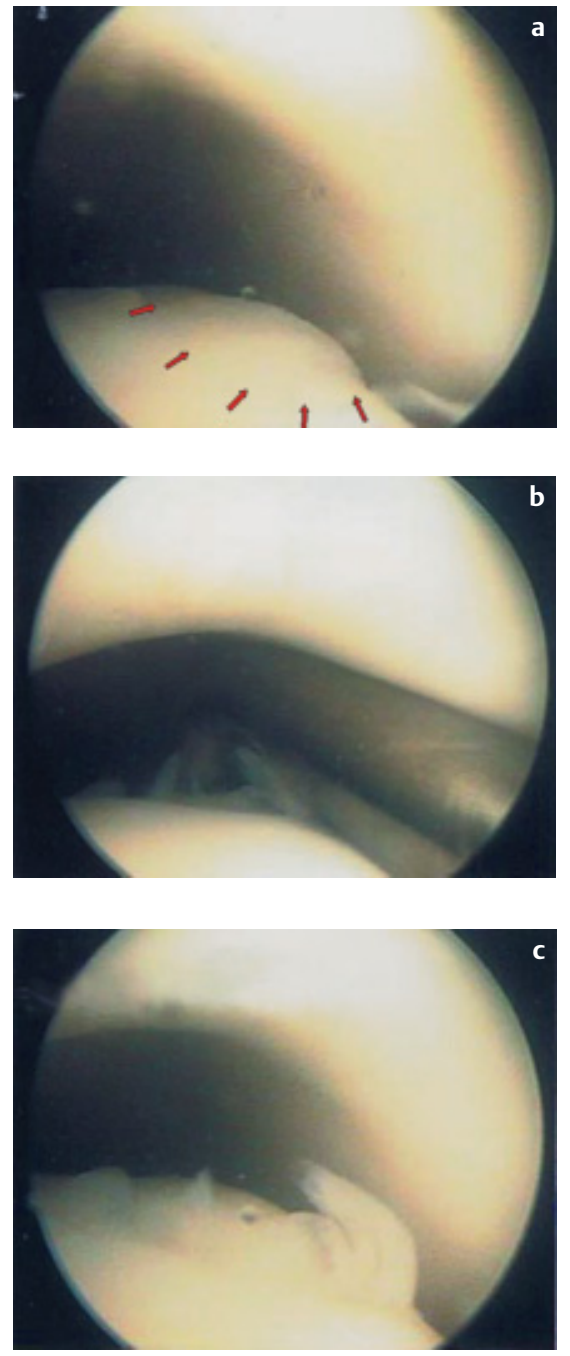

Abb.2a-c Optisch kaum erkennbarer Osteochondrosis-dissecans-Herd (a). Tasthaken versinkt im weichen Knorpel an der medialen Talusrolle (b). Zustand nach ,retrograder“ Anbohrung (c).

\section{Osteophyten}

Osteophyten des oberen Sprunggelenkes finden sich zumeist an der Tibiavorderkante und am ventralen Talus. Häufig sind sie bei Sportlern anzutreffen (Fußballergelenk) [2]. Folge dieser osteophytären Anbauten sind die Einschränkung der Beweglichkeit sowie schmerzhafte, therapieresistente Reizzustände im Bereich der ventralen Gelenkkapsel. Anders als der Röntgenbefund vermuten lässt, zeigt sich in der arthroskopischen Untersuchung des oberen Sprunggelenkes häufig ein deutlich ausgeprägterer Befund. Bei entsprechender Anamnese mit lang anhaltenden Beschwerden ist auch bei nicht eindrucksvollem Röntgenbefund die Indikation zur Arthroskopie gegeben [8].
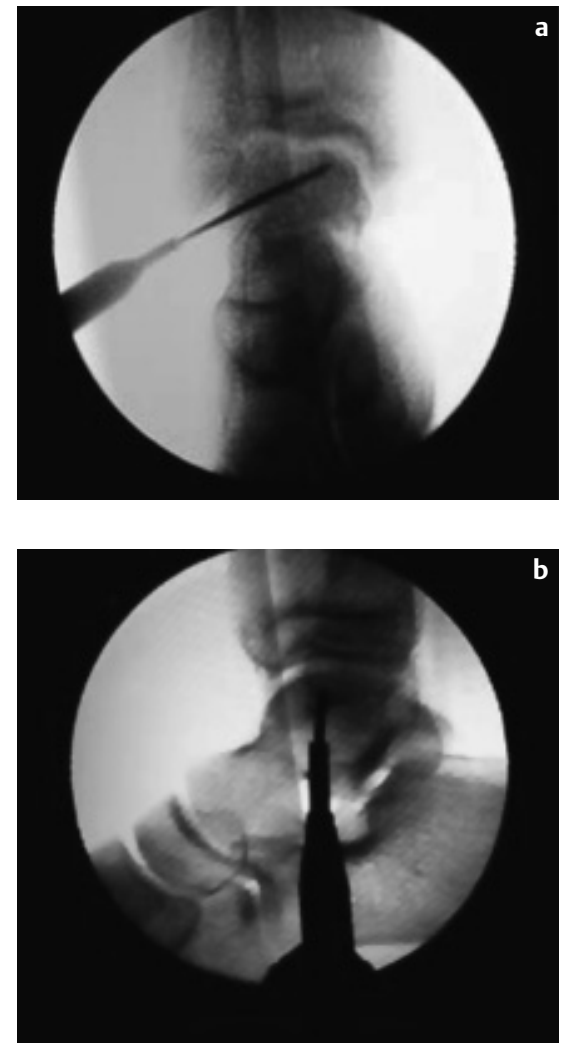

Abb.3au.b Radiologisch kontrollierte „retrograde" Bohrung des Osteochondrosis-dissecans-Herdes. A. p. Ansicht (a) und seitliche Darstellung (b).

\section{Synoviale Reizzustände}

Bei rheumatischen Synovialitiden kommt es zur Ausbildung einer villonodulären Synovitis. Diese führt zu rezidivierenden Gelenkergüssen und persistierenden Kapselschwellungen. Bewegungseinschränkungen infolge eingeklemmter, verdickter Synovia sind die Folge [5]. Durch die arthroskopische Revision kann neben der therapeutischen Synovektomie die Diagnose durch Gewebeentnahme gesichert werden.

\section{Chronische Bandinstabilität}

Die Diagnose der chronischen fibularen Kapselbandinstabilität ist primär anhand der Beschwerden und dem Untersuchungsbefund klinisch zu stellen [8].

Trotzdem empfiehlt sich vor Durchführung einer Kapsel-Band-Plastik die Arthroskopie. Diese erfasst intraartikuläre Veränderungen und gravierende Knorpelschäden. So können Vernarbungen nach rezidivierenden Distorsionen durchtrennt werden. Das führt zu einer deutlichen Besserung der Beschwerden. 
Tab. 2 Mögliche peri- und postoperative Komplikationen der OSG-Arthroskopie.

$$
\begin{aligned}
& \text { a Nervenläsionen } \\
& \text { - Gefäßverletzungen } \\
& \text { - Knorpelverletzungen } \\
& \text { a synoviale Fisteln } \\
& \text { - Sehnenverletzung } \\
& \text { a Gelenkinfektion }
\end{aligned}
$$

Anschließend erfolgt die Stabilisierung des fibularen Kapselbandapparates durch eine offene Operation [9].

\section{Komplikationen der Arthroskopie}

Trotz der geringen Morbidität des Eingriffes ist die Operation nicht ohne Risiko (siehe Tab. 2). Die Angaben zur Komplikationsrate in der Literatur schwanken beträchtlich. Während in einigen Studien von Komplikationen in über $15 \%$ der Fälle berichtet wird, erwähnen andere Autoren eine Komplikationsrate von 0,5-0,6\%. Es werden Nerven-, Knorpel- und Gefäßverletzungen beschrieben [4]. Als eher seltene Komplikationen werden Infektionen und synoviale Fisteln an den Hautschnitten als Folge arthroskopischer Operationen am oberen Sprunggelenk erwähnt.

\section{Kontraindikation}

Frische Verletzungen mit massiven Weichteilschwellungen und Ödeme am oberen Sprunggelenk sowie arteriovenöse Verschlusserkrankungen im fortgeschrittenen Stadium stellen relative Kontraindikationen dar [8]. Darüber hinaus gelten die allgemeinen Kontraindikationen einer Operation auch für ein arthroskopisches Vorgehen.

\section{Technik der Arthroskopie am oberen Sprunggelenk}

\section{Instrumentarium}

Für eine Sprunggelenksarthroskopie wird die gleiche technische Ausstattung benötigt wie für die Spiegelung anderer Gelenke [3] (siehe Tab.3). Diese beinhaltet eine Videokette mit Lichtquelle und Bilddokumentation sowie manuelle oder instrumentelle Distraktion und arthroskopische Operationsinstrumente. Es empfiehlt sich aufgrund der Dimension der Gelenke kleineres Instrumentarium zu verwenden. Aufgrund der kurzen intraartikulären Distanzen ist insbesondere ein kurzer Arthroskopieschaft sinnvoll (siehe Abb.5). Dieser reduziert die Pendelbewe-
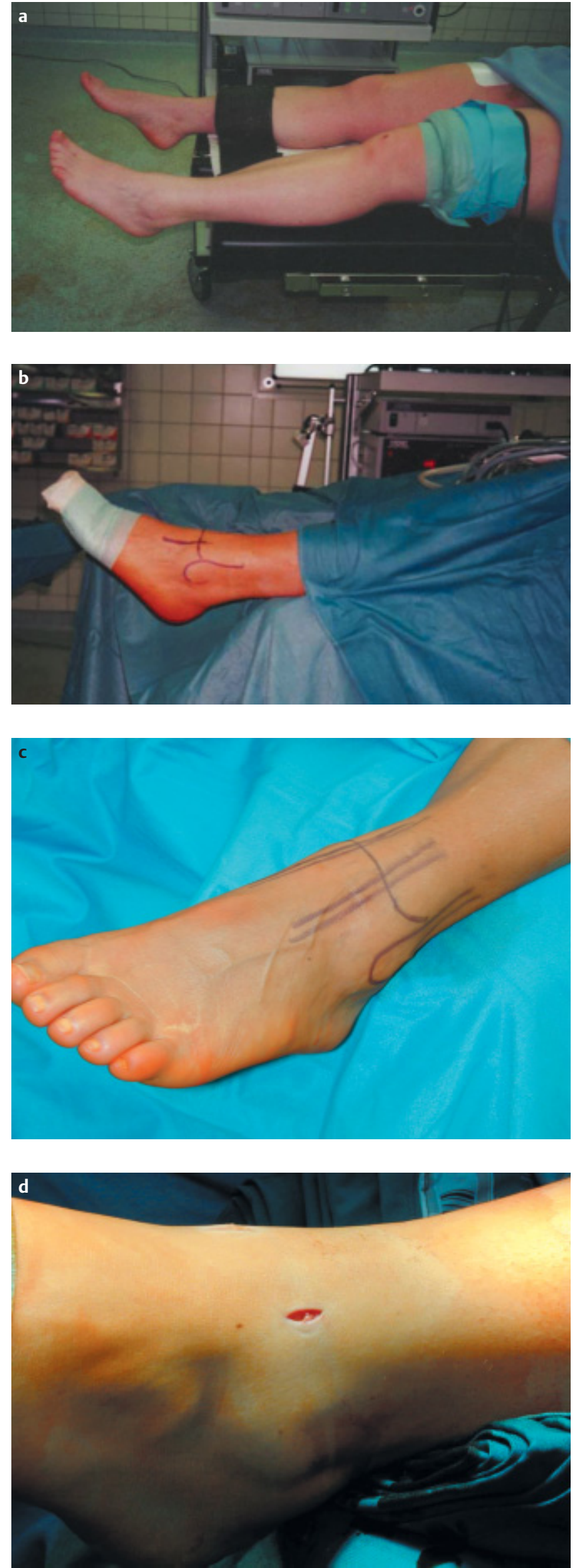

Abb.4a-d Präoperative Lagerung mit freihängendem Fuß (a). Sterile Abdeckung und Kennzeichnung der wichtigen anatomischen Orientierungspunkte (b, c). Anzeichnen anatomischer Leitstrukturen wie Innen- und Außenknöchel und die Tibialis-anterior-Sehne (c). Anschließend Anlage des anterolateralen Standardzugangs (d). 
Tab. 3 Für die effiziente OSG-Arthroskopie notwendiges Instrumentarium.

a Videokette mit Lichtquelle

- Bilddokumentation

n Pumpsystem/Deckenlift

- kurze Optik und Arthroskopieschleuse

- Distraktor

a Tasthaken

- Fasszangen, Punches

- Shaversystem inkl. Cutter und Synovektor

- Elektrochirurgisches Instrumentarium (z. B. Hochfrequenzmesser)

- [Laserinstrumentarium]

gungen des Arthroskopes und schafft somit ein deutlich ruhigeres Bild. Mechanische Instrumente, wie z.B. Fasszangen oder Punches, werden eingesetzt. Als motorbetriebene Instrumente eignen sich Shaversysteme und als elektrochirurgische Instrumente, $z$. B. das Hochfrequenzmesser. Eventuell kommt der Einsatz von Laserinstrumentarium infrage. Es wird im flüssigen Medium arthroskopiert. Als Spülflüssigkeit dient eine elektrolytfreie Lösung (z.B. Purisole). Für ausreichenden Füllungsdruck innerhalb des Gelenkes sorgt entweder die Schwerkraft (Deckenlift) oder ein Pumpsystem [8].

\section{Vorbereitung}

Der Eingriff kann in Leitungsanästhesie oder Vollnarkose durchgeführt werden [3]. Der Patient liegt in Rückenlage und der zu operierende Fuß sollte etwa eine Hand breit über den Operationstisch hinausragen, um eine optimale Plantarflexion zu ermöglichen. Spezielle Beinhalter oder andere Fixationstechniken sind

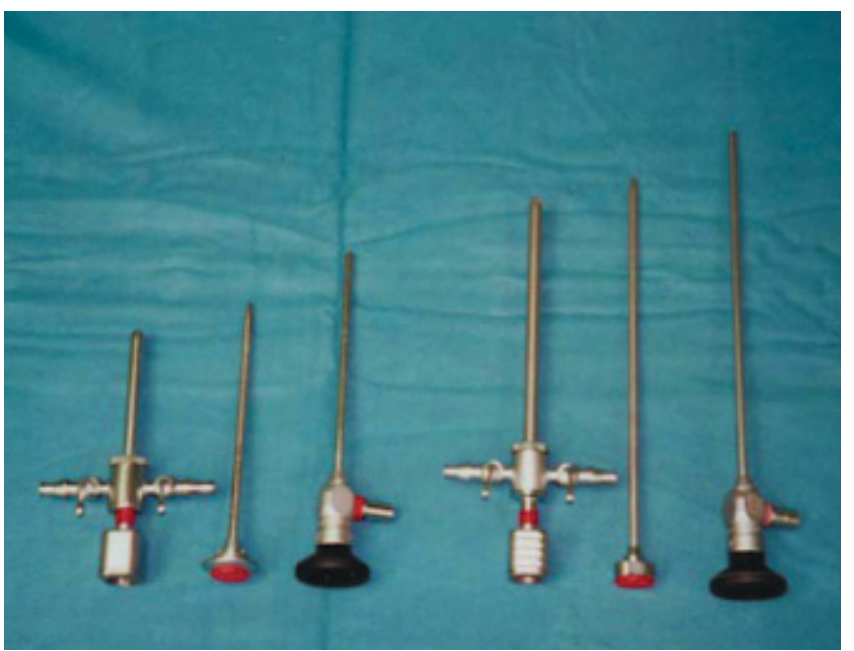

nicht nötig. Eine Druckmanschette wird am Oberschenkel angelegt, auch wenn die Arthroskopie möglichst ohne Blutsperre erfolgen sollte. Nach der chirurgischen Desinfektion wird der Fuß bis zur Unterschenkelmitte frei beweglich abgedeckt. Die Zehen werden gesondert abgeklebt.

Da in flüssigem Medium arthroskopiert wird, sind eine wasserdichte Abdeckung des Operationsgebietes und entsprechend Kittel für den Operateur sinnvoll. Um größere Flüssigkeitsansammlungen und Pfützen zu vermeiden, kann ein Bodensaugsystem eingesetzt werden.

\section{Zugangswege}

Für die Arthroskopie des oberen Sprunggelenkes sind sowohl anteriore als auch posteriore, transmalleoläre sowie transtalare Zugänge beschrieben [3]. Um die häufigsten arthroskopischen Eingriffe durchführen zu können, sind in der Regel der anterolaterale und anteromediale $\mathrm{Zu}$ gang ausreichend. Vor Anlage der $\mathrm{Zu}$ gangswege erfolgt zunächst die Orientierung am Sprunggelenk. Hierzu werden die knöchernen Strukturen getastet. Die Malleolengabel dient als wichtigste Orientierungshilfe. Die Spitze des lateralen Malleolus liegt 1,5 bis $2 \mathrm{~cm}$ distal und im Vergleich zum Innenknöchel etwas dorsal. Der anteriore Gelenkspalt befindet sich ca. $2 \mathrm{~cm}$ proximal der Innenknöchelspitze bzw. etwa $3-4 \mathrm{~cm}$ proximal der Spitze des lateralen Malleolus. Darüber hinaus lassen sich die Sehnenstrukturen und Blutgefäße identifizieren [1].

\section{Anteriore Zugangswege}

Im anterioren Gelenkbereich verlaufen Hautnerven und Blutgefäße ebenso wie

die Strecksehnen. Unter passiver Mobilisation sind die Sehnen leicht zu identifizieren und bieten gute Orientierungspunkte. Entscheidende Leitstrukturen sind die Sehnen des M. extensor hallucis longus, des $\mathrm{M}$. tibialis anterior sowie des M. extensor digitorum longus (siehe Abb. 6b). In unmittelbarer Nachbarschaft dieser Strukturen tastet man bei Bewegung die anterolaterale Talusrolle sowie den anterolateralen Gelenkspalt. Der Nervus saphenus verläuft gemeinsam mit der Vena saphena magna (siehe Abb. 6a) vor dem Innenknöchel und medial der Sehne des Musculus extensor hallucis longus (siehe Abb.6b). Die A. dorsalis pedis mit ihren Begleitvenen befindet sich unter den Strecksehnen der Zehen ebenso wie der Nervus peronaeus profundus. Dieser Bereich sollte bei Anlage eines Zuganges unbedingt umgangen werden.

\section{Anteromedialer Zugang}

Medial der Sehne der M. tibialis anterior wird der anteromediale Zugang angelegt. Er eignet sich zur Inspektion des anterolateralen Gelenkraumes (siehe Abb. 7b). Bei diesem Zugang sind die V. saphena magna und der N. saphenus gefährdet.

\section{Anterolateraler Zugang}

Dieser Zugang dient als Standardzugang (Arthroskopzugang), und wird zuerst angelegt. Er befindet sich lateral der Sehne des $M$. extensor digitorum longus auf Höhe des Gelenkspaltes etwa $3 \mathrm{~cm}$ proximal der Außenknöchelspitze (siehe Abb. 6b). Die V. saphena parva sowie oberflächliche Hautäste des N. peronaeus superficialis können hier verletzt werden.

Der anterolaterale Zugang ist der Standardzugang.

Abb. 5 Arthroskopieschleuse für das obere Sprunggelenk. Die Instrumente sind kürzer als die, die zur Kniegelenksarthroskopie verwendet werden.

\section{Anterozentraler Zugang}

Sehr oft werden anterozentrale Zugänge beschrieben. Diese Zugänge sind gefährlich, da A. tibialis anterior und der N. peroneus profundus sowie Hautvenen verletzt werden können.

Der anterozentrale Zugang ist gefährlich.

\section{Tiefer anterolateraler Zugang}

Dieser Zugang wird als akzessorischer Zugang beschrieben. Er liegt wenige $\mathrm{mm}$ proximal und etwa $1 \mathrm{~cm}$ vor der Fibulaspitze. Er dient zur Beurteilung der Fibulaspitze und der davon distal liegen- 

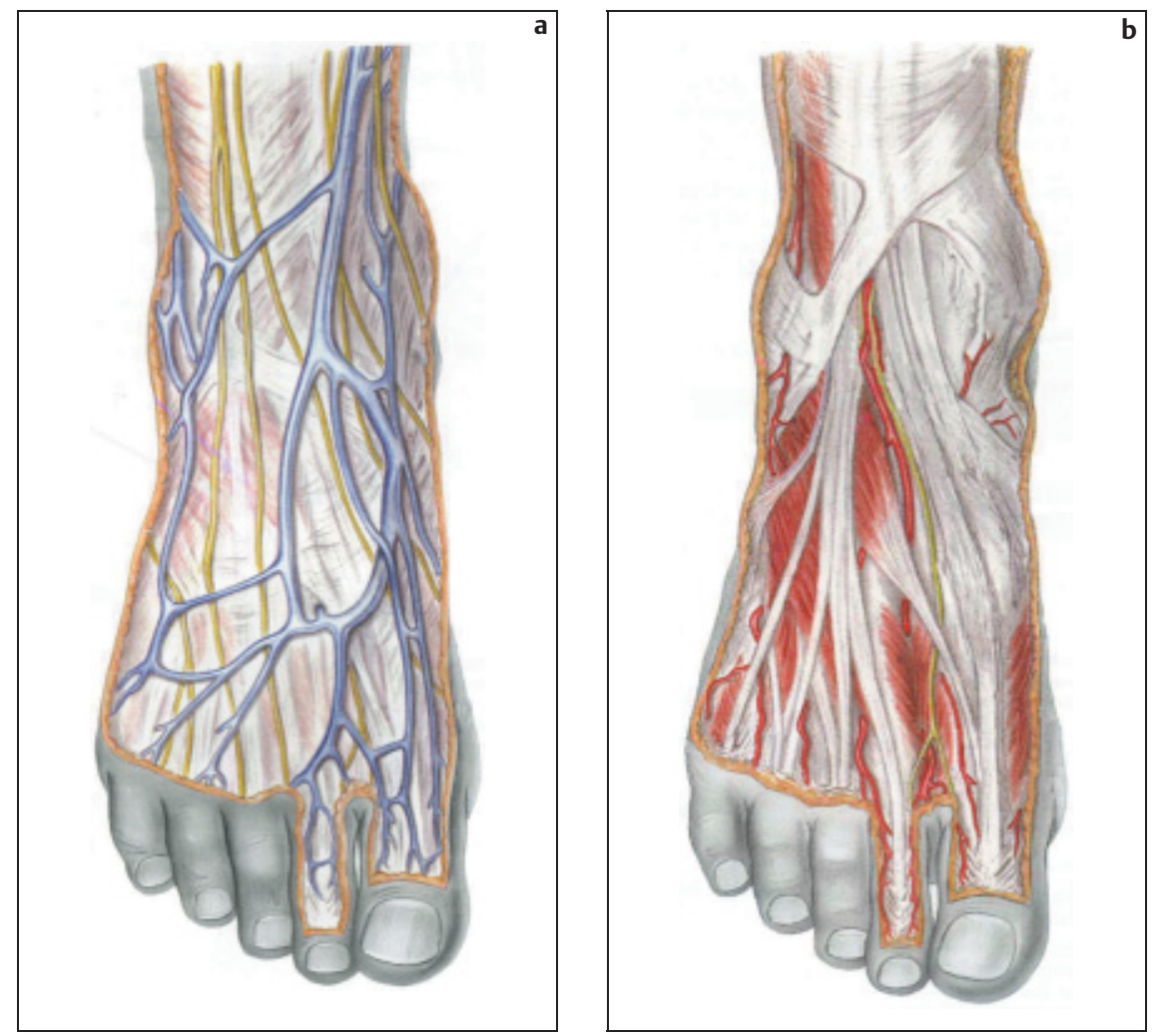

Abb.6au.b Anatomische Strukturen im Bereich des Fußrückens. Gefährdete Nerven und Gefäße (a). Arterielle Gefäße (b).
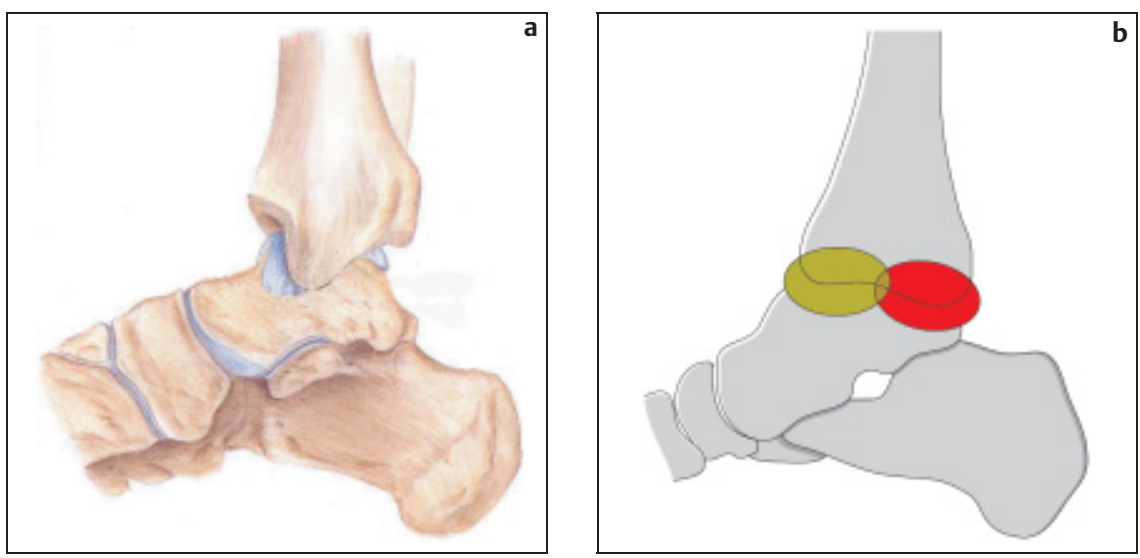

Abb. 7au.b Oberes Sprunggelenk in der seitlichen Ansicht (a). Die Gelenkbereiche, die vom ventralen Zugang (grün) und vom dorsalen Zugang (rot) aus inspiziert werden können.

den Strukturen wie z.B. fixierte Gelenkkörper.

\section{Tiefer anteromedialer Zugang}

Ebenso wie der tiefe anterolaterale $\mathrm{Zu}-$ gang ist dies ein akzessorischer Zugang. Er liegt $1 \mathrm{~cm}$ vor der Vorderkante des Innenknöchels.

Die anterioren Zugänge reichen zumeist zur Beurteilung der pathologischen Befunde.
Dieser als posteriorer Standardzugang salen Talusrolle (siehe Abb. 7b).

\section{Transtendinöser Zugang}

Als zweiter posteriorer Zugang kann der transtendinöse Zugang angelegt werden.
Er wird in der Mitte der Achillessehne platziert.

\section{Operationstechniken}

Nach Markierung der geplanten Zugänge, des Gelenkspaltes und der tastbaren knöchernen Strukturen mit einem Hautmarker (siehe Abb. 4c) erfolgt die Anlage des anterolateralen Zugangs (siehe Abb. 4d). Eine Injektionskanüle wird über den anterolateralen Zugang in den Intraartikulärraum vorgeschoben. Das Erreichen des Gelenkraumes ist dadurch gekennzeichnet, dass die Nadel problemlos nach medial und lateral geschwenkt werden kann. Jetzt wird eine Spritze aufgesetzt und anschließend erfolgt die Auffüllung des Gelenkes mit Flüssigkeit. Das Gelenk fasst etwa $15 \mathrm{ml}$ Flüssigkeit. Nach Injektion der ersten $5 \mathrm{ml}$ wird die Spritze von der Kanüle abgenommen. Entleert sich die Spülflüssigkeit tropfenweise oder im Strahl, ist die intraartikuläre Lage der Injektionskanüle gesichert. Wenn die Injektion der Spülflüssigkeit nur gegen einen starken Widerstand möglich ist, liegt die Kanüle entweder extraartikulär oder der Gelenkspalt ist durch straffes Narbengewebe verlötet. Nach Korrektur der Lage der Injektionskanüle wird das Gelenk weiter aufgefüllt. Mit zunehmender Gelenkfüllung kommt es zu einer Dorsalflexion des Fußes und Kapselschwellung im Bereich des medialen und lateralen Gelenkspaltes. Nach Zurückziehen der Kanüle wird die Haut auf eine Länge von $1-2 \mathrm{~cm}$ mit einem spitzen Skalpell durchtrennt. Dann erfolgt die stumpfe Präparation des Subkutangewebes mit einer kleinen Klemme ohne die Gelenkkapsel zu eröffnen. Anschließend wird die Arthroskopieschleuse mit eingesetztem Trokar unter rotierenden Bewegungen auf die Kapsel vorgeschoben. Man spürt das Erreichen des Intraartikulärraumes an dem plötzlich nachlassenden Widerstand. Jetzt wird die Optik in die Arthroskopieschleuse eingeführt. Es folgt die Inspektion.

Die vordere Gelenkkapsel und der anteromediale Gelenkraum lassen sich vom anterolateralen Zugang aus gut einsehen (siehe Abb. 7b).Zur Palpation intraartikulärer Veränderungen wird ein Tasthaken benötigt. Dies macht die Anlage eines Instrumentenzugangs notwendig. Er wird im Bereich der anteromedialen Kapsel unter arthroskopischer Kontrolle angelegt. Die durchscheinende Lichtquelle erlaubt die Beurteilung der subkutan verlaufenden Sehnen und Gefäße. Unter 
Schonung dieser Strukturen wird eine weitere Injektionskanüle in das Gelenk eingestochen. Die ideale Lage des $\mathrm{Zu}-$ gangs ist in Höhe des Gelenkspaltes. Jetzt kann der Tasthaken eingeführt werden. Er dient zur Beurteilung der Knorpelflächen und anderer intraartikulärer Veränderungen sowie zur Mobilisation und Retraktion von Synoviafalten und Narbensträngen. Bei starken intraartikulären Verwachsungen kann durch Resektion der Narben mit Hilfe des Shavers die Sicht verbessert werden. Dieser kann ebenfalls über diesen Zugang in das Gelenk eingeführt werden.

Zur ausreichenden Inspektion des Gelenkes ist der Wechsel des Zuganges für die Optik und Instrumente notwendig. Hierbei kann ein Wechselstab eingesetzt werden.

Nach Platzieren der Optik über den anteromedialen Zugang lassen sich die ventralen Kapselstrukturen und der anterolaterale Gelenkraum gut einsehen (siehe Abb. 7b). Bei zu erwartenden pathologischen Veränderungen im Bereich der posterioren Gelenkanteile ist die Anlage von weiteren posterioren Zugängen notwendig. Auch diese werden in der Kanülentechnik angelegt. Es wird zuerst der posterolaterale Zugang angelegt. Nach Vorschieben des Arthroskopes auf die dorsale Kapsel, erfolgt die Anlage des Zugangs unter Sicht [8].

\section{Untersuchungsgang}

Zunächst wird das anteriore Kompartment über den anterolateralen Zugang inspiziert. Hierbei können die Tibiavorderkante und die zwei Drittel der vorderen Talusgelenkfläche und die anteriore Kapsel beurteilt werden (siehe $\mathbf{A b b}$. 7b). Anschließend erfolgt die Inspektion des anteromedialen Kompartments mit der medialen Taluskante sowie dem Innenknöchel und der medialen Taluswange [7]. Dann erfolgt der Zugangswechsel. Das Arthroskop wird unter Zuhilfenahme des Wechselstabes in den medialen Instrumentenzugang eingeführt. Dadurch lässt sich der laterale Teil des anterioren Kompartments mit der außenseitigen Talus- und Tibiavorderseite beurteilen. Die Syndesmose ist in einigen Fällen ebenfalls einzusehen. Bei weit mehr als $90 \%$ aller Sprunggelenksarthroskopien ist die Anlage der anterioren Zugangswege ausreichend. Nur bei Verdacht auf eine intraartikuläre Veränderung im posterioren Gelenkraum muss auch ein posteriorer Zugang angelegt werden. Über den post-
Tab. 4 Operative Techniken der Arthroskopie am oberen Sprunggelenk.

- Spülung

— Entfernung freier Gelenkkörper

- partielle/komplette Synovektomie

- diagnostische Gewebeentnahme

- Durchtrennung von Narbengewebe mit HF-Messer oder Shaversystem

- Arthrolyse

- Refixation gelöster Knorpelareale

- Microfracture Technik

- subchondrale Abrasionschondroplastik

- Debridement instabiler Knorpelanteile

- anterograde/retrograde Anbohrung

- osteochondrale Transplantation

- Abtragung von osteophytären Ausziehungen

- Arhrodese

erolateralen Zugang lassen sich das posteromediale Kompartment und der dorsale Gelenkbereich ausreichend beurteilen (siehe Abb. 7b).

Nach Inspektion erfolgt die Palpation der intraartikulären Veränderung mit dem Tasthaken. Anschließend erfolgt eine notwendige operative Therapie.

\section{Therapeutische Möglichkeiten}

Die Möglichkeiten der arthroskopischen Therapie sind groß (siehe Tab.4). Sie reichen von einfachen Entfernungen freier Gelenkkörper durch Fasszangen bis hin zu aufwendigen Rekonstruktionen an den Knorpelflächen mit Induktion von Faserknorpelregeneraten durch Abrasion oder retrograde Knorpelbohrungen [3] Darüber hinaus werden auch KnorpelKnochen-Transplantationen und Arthrodesen arthroskopisch beschrieben [6] Abtragung von Osteophyten oder Entfernen erkrankter Synovia werden ebenfalls durchgeführt [8]. Auch partielle oder subtotale Synovektomien bei Erkrankungen aus dem rheumatischen Formenkreis können arthroskopisch vorgenommen werden [5].

\section{Schlussfolgerung}

Die technische Weiterentwicklung der Sprunggelenksarthroskopie hat sie zu einem etablierten chirurgischen Standardverfahren werden lassen [6]. In gleichem Maße wie sich das Spektrum der Indikationen vergrößert hat, hat die Anzahl der therapeutischen Eingriffe zuge- nommen. Voraussetzungen zur erfolgreichen Arthroskopie am oberen Sprunggelenk ist zunächst eine korrekte klinische Diagnostik unter Einsatz der bildgebenden apparativen Diagnostik einschließlich MRT [8]. Die engen anatomischen Verhältnisse erfordern größte Sorgfalt und die Einhaltung operativer Standards, um peri- und postoperative Komplikationen zu vermeiden [9]. Ein standardisierter Untersuchungsgang garantiert die umfassende Exploration des Gelenkes und die Therapie der pathologischen Befunde in allen Gelenkanteilen. Somit hat sich ein Operationsverfahren etabliert, was dem Patienten bei geringer operationsinduzierter Morbidität einen maximalen Therapieerfolg garantiert.

\section{Literatur}

${ }^{1}$ Andrews JR, Previte WJ, Carson WG. Arthroscopy of the ankle: technique and normal anatomy. Foot Ankle 1985; 6: 129-133

${ }^{2}$ Attmanspacher W, Dittrich V, Schätzler A, Stedtfeld HW. Arthroskopie des oberen Sprunggelenks beim „Fußballergelenk“. Arthroskopie 1998; 11: 203-209

${ }^{3}$ Benedetto KP, Glötzer W. Indikation und Technik der Arthroskopie am oberen Sprunggelenk. Arthroskopie 1991; 4: 9-14

${ }^{4}$ Ferkel RD, Heath DD, Guhl JF Neurological complications of ankle arthroscopy. Arthroscopy 1996; 12: 200-208

${ }^{5}$ Friscia DA. Pigmented villonodular synovitis of the ankle: a case report and review of literature. Foot Ankle Int 1994; 15: 674-678

${ }^{6}$ Jerosch J. Arthroscopic ankle surgery. Indications, surgical technique, results, complications. Orthopäde 1999; 28: [6] 538-549

${ }^{7}$ Landsiedl F. Die Arthroskopie des oberen Sprunggelenks. Arthroskopie 1991; 4: 2-8

${ }^{8}$ Strobel M. Arthroskopische Chirurgie. Springer-Verlag, Berlin Heidelberg New York

9 Unger F, Lajtai G, Ramadani F, Aitzetmüller G, Orthner E. Arthroskopie des oberen Sprungelenks. Unfallchirurg 2000; 103: [10] 858-863

${ }^{10}$ Whipple TL. Meniscoid lesions of the ankle. Clin Sport Med 1991; 10: 661-667

\section{Dr. med. Kai Ruße \\ Assistenzarzt \\ Dr. med. Ralf Müller-Rath \\ Assistenzarzt \\ Dr. med. Axel Pommer \\ Oberarzt \\ Prof. Dr. med. Andreas Dávid \\ Klinikdirektor}

Klinik für Unfall- und Wieder-

herstellungschirurgie

Klinikum Wuppertal GmbH

Heusnerstr. 40

42283 Wuppertal 